\title{
A wide angle view of the Sagittarius dwarf spheroidal galaxy
}

\section{A CEMP-r/s star in the Sagittarius dwarf spheroidal galaxy ${ }^{\star, \star \star}$}

\author{
L. Sbordone ${ }^{1}$, C. J. Hansen ${ }^{2}$, L. Monaco ${ }^{3}$, S. Cristallo ${ }^{4,5}$, P. Bonifacio ${ }^{6}$, E. Caffau ${ }^{6}$, S. Villanova ${ }^{7}$, and P. Amigo ${ }^{8}$ \\ 1 ESO - European Southern Observatory, Alonso de Cordova 3107, Vitacura, Santiago, Chile \\ e-mail: 1sbordon@eso.org \\ 2 Max Planck Institute for Astronomy, Heidelberg, Königstuhl 17, 69117 Heidelberg, Germany \\ 3 Departamento de Ciencias Fisicas, Universidad Andres Bello, Fernandez Concha 700, Las Condes, Santiago, Chile \\ 4 INAF - Osservatorio Astronomico d'Abruzzo, Via Maggini snc, Teramo, Italy \\ 5 INFN - Sezione di Perugia, Via A. Pascoli, Perugia, Italy \\ 6 GEPI, Observatoire de Paris, Université PSL, CNRS, Place Jules Janssen, 92195 Meudon, France \\ 7 Departamento de Astronomía, Universidad de Concepción, Casilla 160-C, Concepción, Chile \\ 8 Facultad de Ingeniería y Ciencias, Universidad Adolfo Ibáñez, Avenida Padre Hurtado 750, Viña del Mar 2520000, Chile
}

Received 8 March 2020 / Accepted 5 May 2020

\section{ABSTRACT}

\begin{abstract}
We report on the discovery and chemical abundance analysis of the first CEMP-r/s star detected in the Sagittarius dwarf spheroidal galaxy (Sgr dSph) by means of UVES high-resolution spectra. The star, found in the outskirts of Sgr dSph, along the major axis of the main body, is a moderately metal-poor giant $\left(T_{\text {eff }}=4753 \mathrm{~K}, \log g=1.75,[\mathrm{Fe} / \mathrm{H}]=-1.55\right)$ with $[\mathrm{C} / \mathrm{Fe}]=1.13$, placing it in the so-called "high-carbon band", and strong $s$-process and $r$-process enrichment $([\mathrm{Ba} / \mathrm{Fe}]=1.4,[\mathrm{Eu} / \mathrm{Fe}]=1.01)$. Abundances of 29 elements from $\mathrm{C}$ to Dy were obtained. The chemical pattern appears to be best fitted by a scenario where an $r$-process pollution event pre-enriched the material out of which the star was born as secondary in a binary system whose primary evolved through the AGB phase, providing C- and s-process enrichment
\end{abstract}

Key words. galaxies: abundances - galaxies: individual: Sgr dSph - Galaxy: abundances - stars: abundances stars: chemically peculiar

\section{Introduction}

Carbon-enhanced metal-poor stars (hereafter CEMP) are objects of low metallicity that show a high abundance of $\mathrm{C}$ relative to iron. A thorough historical perspective of carbon-enhanced stars is given by Caffau et al. (2018), and we refer to that paper and references therein for further details. Beers \& Christlieb (2005) introduced a classification of CEMP stars based on their abundances of neutron-capture elements. In particular, for what is relevant here, the autors defined two classes of stars that appear to be enriched in neutron-capture elements. The authors sorted the stars according to whether only the elements produced in the slow neutron-capture process ( $s$-process) or also the nuclei produced in the rapid neutron-capture process ( $r$-process) were enhanced. Taking into account that most $\mathrm{Ba}$ isotopes are produced in the $s$-process and that the vast majority of Eu nuclei are produced in the $r$-process, Beers \& Christlieb (2005) defined CEMP-s stars as stars that satisfy $[\mathrm{C} / \mathrm{Fe}]>+1.0,[\mathrm{Ba} / \mathrm{Fe}]>$ +1.0 , and $[\mathrm{Ba} / \mathrm{Eu}]>+0.5$ and CEMP-r/s as stars that satisfy $[\mathrm{C} / \mathrm{Fe}]>+1.0$ and $0.0<[\mathrm{Ba} / \mathrm{Eu}]<+0.5$. Lucatello et al. $(2005)$ and later Starkenburg et al. (2014) argued based on repeated radial velocity measurements of CEMP stars that the binary

\footnotetext{
* Line-by-line abundance tables are are only available at the CDS via anonymous ftp to cdsarc.u-strasbg. fr (130.79.128.5) or via http://cdsarc.u-strasbg.fr/viz-bin/cat/J/A+A/641/A135 $\star \star$ Based on data collected with UVES at $8.2 \mathrm{~m}$ VLT-UT2 (Kueyen) telescope under ESO programme 083.B-0774. This paper includes data gathered with the $6.5 \mathrm{~m}$ Magellan Telescopes located at Las Campanas Observatory, Chile.
}

frequency among CEMP-s stars is compatible with a population composed to $100 \%$ of binary stars. Spite et al. (2013) and Bonifacio et al. (2018) pointed out that CEMP stars have a distinctly bi-modal distribution in carbon abundances $\mathrm{A}(\mathrm{C})^{1}$ : stars of the low-carbon band $(\mathrm{A}(\mathrm{C}) \leq 7.6)$ and stars of the highcarbon band $(\mathrm{A}(\mathrm{C})>7.6)$. The high-carbon band is populated almost exclusively by stars of the classes CEMP-s and CEMPr/s. Recently, Arentsen et al. (2019) revised the binary frequency among CEMP stars and estimated a lower frequency than did Lucatello et al. (2005) and Starkenburg et al. (2014), but they confirmed that the binary frequency among stars of the highcarbon band (47\%) is much higher than that of those of the low-carbon band (18\%). The simplest and most widely adopted interpretation of this observational picture is that the high-carbon band stars are the result of mass transfer in a binary system (Abate et al. 2018).

It is straightforward to identify the companion of CEMP-s stars that transferred mass as an asymptotic giant branch (AGB) star, which can produce both the excess carbon and the neutroncapture elements (see, e.g., Cristallo et al. 2011; Bisterzo et al. 2012; Käppeler et al. 2011, and references therein). The situation for CEMP-r/s stars is less clear because the neutron densities required for the $s$-process and the $r$-process differ by at least ten orders of magnitude (see e.g. Hampel et al. 2016, and references therein), so that they are believed to occur in different astrophysical sites. One model for explaining CEMP-r/s stars is to assume that the cloud out of which the star was formed

1 We adopt the notation for any element $\mathrm{X}, \mathrm{A}(\mathrm{X})=\log _{10}(\mathrm{X} / \mathrm{H})+12$. 
has previously been enriched in $r$-process elements (see, e.g., Bisterzo et al. 2012) by a different source, such as a neutronstar merger (Thielemann et al. 2017; Watson et al. 2019), a magnetar (Siegel et al. 2019), or a magnetohydrodynamic (MHD) supernova (Nishimura et al. 2015). Other more contrived scenarios have been proposed, and we refer to Hampel et al. (2016) for a concise summary of the relevant literature. Recently, computations have been made to explore nucleosynthesis at intermediate neutron densities, in the range $10^{7}-10^{15} \mathrm{~cm}^{-3}$ in AGB (Hampel et al. 2016) and rapidly accreting white dwarfs (RAWDs, Denissenkov et al. 2019) to explain the origin of CEMP-r/s stars. The high end of this neutron density regime was originally investigated by Cowan \& Rose (1977) to explain the production of ${ }^{14} \mathrm{C}$ and neutrons in red giants. Now it is commonly referred to as the $i$-process.

In this paper we report the discovery and analysis of the first CEMP-r/s star found in the Sgr dSph galaxy. The significance of this discovery is that we are able to compare the properties of this particular class of stars to those of the Galactic stars. Such a comparison could give information on how the Galactic environment affects, or does not affect, the evolution of these exceptional objects.

\section{Observations}

The star GIU J190734.24-315102.1 (henceforth J1907) was catalogued as part of the Sgr dSph Wide Angle survey (Giuffrida et al. 2010; Hansen et al. 2018a; Sbordone et al. 2015). Giuffrida et al. (2010) detected it as a probable Sgr dSph member from VLT-VIMOS ${ }^{2}$ (Le Fèvre et al. 2003) $V$ and $I$ photometry and low-dispersion spectroscopy. Subsequently, it was followed up with FLAMES ${ }^{3}$-Giraffe (Pasquini et al. 2000), which allowed us to firmly establish it as a high-probability member and to derive an overall metallicity of $[\mathrm{Fe} / \mathrm{H}] \sim-1.5$ (Sbordone et al., in prep.). Finally, together with a group of low-metallicity Sgr dSph member stars, it was re-observed with UVES $^{4}$ (Dekker et al. 2000) to obtain high-resolution highquality spectra for a detailed chemical analysis. The other stars that were re-observed with UVES-slit together with J1907 have been presented in Hansen et al. (2018a).

Coordinates, photometry, proper motions, and atmospheric parameters for $\mathrm{J} 1907$ are listed in Table 1. Coordinates and $V, I$ magnitudes come from Giuffrida et al. (2010). J1907 is situated in the easternmost major-axis field described in Giuffrida et al. (2010), Sgr4, roughly 3 degrees away from the center of NGC 6715 (M54), which coincides with the center of the Sgr $\mathrm{dSph}$. While its radial velocity differs from that of NGC 6715 (143.06 $\mathrm{km} \mathrm{s}^{-1}$, Baumgardt et al. 2019) by roughly the amount of the cluster velocity dispersion $\left(10.5 \mathrm{~km} \mathrm{~s}^{-1}\right.$, Harris 1996, 2010 revision) and its metallicity is quite close to that of the cluster, its distance is vastly larger than the estimated NGC 6715 tidal radius $\left(7.5^{\prime}\right.$, Trager et al. 1995). It is therefore unlikely that the star originated within NGC6715.

This work is mainly based on the analysis of the UVESslit spectra of J1907, observed in two 3005s exposures starting on 2009 April 25, 07:28:27 and 08:25:22 UT. The spectra were obtained in dichroic 1 mode, with central wavelengths of $390 \mathrm{~nm}$ and 580nm in the UVES blue and red arm respectively, at a low airmass (1.13-1.01). The slit was set to $1.4^{\prime \prime}$ on both arms, but seeing conditions were excellent $\left(\sim 0.45^{\prime \prime}\right)$, thus increasing the

\footnotetext{
2 VLT: Very Large Telescope; VIMOS: VIsual Multi Object Spectrograph.

3 FLAMES: Fibre Large Array Multi Element Spectrograph.

4 UVES: UV-Visual Echelle Spectrograph.
}

Table 1. Coordinates and parameters for J1907.

\begin{tabular}{lc}
\hline \hline GIU J190734.24-315102.1 \\
\hline RA (J2000) & \\
Dec (J2000) & $19 \mathrm{~h} \mathrm{07m} \mathrm{34.23s}$ \\
Mag $(V, I)$ & $-31 \mathrm{deg} 50^{\prime} 59.2^{\prime \prime}$ \\
$V_{\text {rad }}$ & $17.742,15.466$ \\
pm $(\mathrm{RA} *, \mathrm{Dec})$ & $155.04 \pm 0.35 \mathrm{~km} \mathrm{~s}^{-1}$ \\
$T_{\text {eff }}$ & $-2.65 \pm 0.13,-1.32 \pm 0.12 \mathrm{mas} \mathrm{yr}^{-1}$ \\
$\log g$ & $4753_{-160}^{+180} \mathrm{~K}^{-2}$ \\
$V_{\text {turb }}$ & $1.75 \pm 0.1 \mathrm{~cm} \mathrm{~s}^{-2}$ \\
{$[\mathrm{Fe} / \mathrm{H}]$} & $1.51 \pm 0.2 \mathrm{~km} \mathrm{~s}^{-1}$ \\
\hline
\end{tabular}

Notes. Internal parameter errors are quoted (see Appendix A). Magnitudes and coordinates come from Giuffrida et al. (2010), proper motions have been derived from Gaia DR2.

resolution to about $R=60000$. Our analysis is based on scienceready reduced spectra released by $\mathrm{ESO}^{5}$. Coadding the two spectra, a signal-to-noise ratio $(\mathrm{S} / \mathrm{N})$ of $\sim 80$ per sample $(1 \times 1$ binning $)$ around $630 \mathrm{~nm}$ is reached.

An additional spectrum was obtained on 2015 August 21 (23:22:55 UT) with the MIKE ${ }^{6}$ spectrograph at Magellan Clay telescope in Las Campanas observatory, in Chilean time. The single 1200 s spectrum covers the $483-916 \mathrm{~nm}$ range and was taken with the $0.7^{\prime \prime} \times 5^{\prime \prime}$ slit in mediocre transparency conditions for the purpose of searching for radial velocity variations, and has an $S / N \sim 15$ per sample $(2 \times 2$ binning $)$ around $650 \mathrm{~nm}$.

Radial velocities were determined by cross-correlating the spectra against a synthetic template of similar atmospheric parameters, employing the red arm spectra in the UVES case. The two UVES spectra gave 155.0 and $154.6 \pm 0.7 \mathrm{~km} \mathrm{~s}^{-1}$, respectively. The MIKE spectra yield $155.3 \pm 0.5 \mathrm{~km} \mathrm{~s}^{-1}$. As a consequence, there is no evidence that the star shows significant radial velocity variations. In Table 1 the weighted mean of the three measurements is given as reference. The radial velocity is compatible with a membership with Sgr dSph. Bellazzini et al. (2008) derived a radial velocity for the core of Sgr dSph (up to 9' from the center) of $141 \mathrm{~km} \mathrm{~s}^{-1}$, with a dispersion of roughly $10 \mathrm{~km} \mathrm{~s}^{-1}$. Majewski et al. (2013), covering larger distances from the center (up to about 2.5 degrees), detected a significant increase in velocity dispersion with increasing distance from the galaxy core, which is more pronounced for the more metal poor population. J1907 is even farther away from the Sgr dSph center, at the extreme of the covered range along the galaxy major axis, and its velocity is well compatible with the other Sgr dSph members observed in the same field (Sbordone et al., in prep.). Proper motions, as derived from Gaia DR2, also confirm a membership in Sgr dSph: J1907 shows $\mu=$ $-2.654,-1.323$ (RA, Dec, mas/year), versus a Sgr dSph average of -2.692,-1.359 (Gaia Collaboration 2018). Because the Sgr dSph proper motions are very tightly clustered, J1907 can be considered a bona fide member of Sgr dSph.

\section{Parameter determination and abundance analysis}

Stellar parameters (listed in Table 1) were determined spectroscopically by means of the MyGIsFOS automated abundance analysis code (Sbordone et al. 2014). The employed grid of

\footnotetext{
5 http://archive.eso.org/wdb/wdb/adp/phase3_spectral/ form

6 MIKE: Magellan Inamori Kyocera Echelle.
} 
Table 2. Atomic data and $[\mathrm{X} / \mathrm{Fe}]$ for lines measured manually.

\begin{tabular}{|c|c|c|c|}
\hline $\begin{array}{l}\text { Wavelength } \\
(\mathrm{nm})\end{array}$ & $\begin{array}{c}\text { lower e. } \\
(\mathrm{eV})\end{array}$ & $\log g f$ & {$[\mathrm{X} / \mathrm{Fe}]$} \\
\hline \multicolumn{4}{|l|}{ Sr II } \\
\hline 4077.714 & 0.000 & 0.15 & 0.47 \\
\hline \multicolumn{4}{|l|}{ Y II } \\
\hline 485.4867 & 0.992 & -0.380 & 0.55 \\
\hline 488.3684 & 1.083 & 0.070 & 0.45 \\
\hline 520.5731 & 1.032 & -0.340 & 0.40 \\
\hline \multicolumn{4}{|l|}{$\mathrm{Zr} \mathrm{I}$} \\
\hline 535.0851 & 2.322 & -0.590 & 0.95 \\
\hline \multicolumn{4}{|l|}{ Zr II } \\
\hline 496.2310 & 0.971 & -2.000 & 0.90 \\
\hline \multicolumn{4}{|l|}{$\mathrm{Ba}$ II } \\
\hline 585.3686 & 0.604 & -2.066 & 1.40 \\
\hline \multicolumn{4}{|l|}{ La II } \\
\hline 4970.383 & 0.321 & -1.683 & 1.30 \\
\hline 4986.765 & 0.173 & -2.300 & 1.25 \\
\hline 5259.380 & 0.173 & -1.950 & 1.30 \\
\hline \multicolumn{4}{|l|}{$\mathrm{Ce} \mathrm{I}$} \\
\hline 524.9605 & 0.410 & -0.630 & 1.40 \\
\hline \multicolumn{4}{|l|}{$\mathrm{Ce}$ II } \\
\hline 488.2463 & 1.527 & 0.190 & 1.30 \\
\hline 489.3952 & 1.326 & -0.538 & 1.30 \\
\hline 491.4924 & 0.879 & -0.810 & 1.15 \\
\hline 518.7458 & 1.211 & 0.170 & 1.30 \\
\hline 523.7067 & 1.319 & -0.620 & 1.30 \\
\hline \multicolumn{4}{|l|}{ Pr II } \\
\hline 525.9614 & 0.633 & -3.727 & 1.30 \\
\hline 532.2710 & 0.482 & -1.878 & 1.20 \\
\hline 538.1260 & 0.508 & -0.461 & 1.20 \\
\hline \multicolumn{4}{|l|}{$\mathrm{Nd}$ II } \\
\hline 406.1080 & 0.471 & 0.550 & 1.30 \\
\hline 485.9030 & 0.320 & -0.440 & 1.50 \\
\hline 487.6110 & 0.559 & -1.230 & 1.30 \\
\hline 488.2880 & 0.742 & -1.410 & 1.20 \\
\hline 494.2960 & 0.742 & -1.130 & 1.30 \\
\hline 516.7920 & 0.559 & -1.180 & 1.30 \\
\hline 517.6780 & 1.120 & -0.840 & 1.30 \\
\hline 518.1169 & 0.859 & -0.600 & 1.17 \\
\hline 525.5510 & 0.204 & -0.670 & 1.50 \\
\hline \multicolumn{4}{|l|}{ Sm II } \\
\hline 4913.260 & 0.659 & -0.93 & 1.28 \\
\hline 4948.630 & 0.543 & -0.95 & 1.30 \\
\hline 4952.370 & 0.333 & -1.25 & 1.06 \\
\hline \multicolumn{4}{|l|}{ Gd II } \\
\hline 517.6290 & 1.059 & -0.710 & 1.15 \\
\hline \multicolumn{4}{|l|}{ Dy II } \\
\hline 516.9690 & 0.100 & -1.950 & 1.20 \\
\hline
\end{tabular}

synthetic spectra was the same as used in Sbordone et al. (2015), and was based on ATLAS12 1D-LTE atmospheric models and SYNTHE synthetic spectra (Kurucz 2005; Sbordone et al. 2004; Sbordone 2005; Castelli 2005). The input list of features passed to MyGIsFOS was also used in Sbordone et al. (2015), but the code may have kept a different subset of them. Because of the high $\mathrm{C}$ abundance and because MyGIsFOS is not designed to derive precise abundances for elements that strongly deviate from a solar-scaled composition (see Sbordone et al. 2014, for details), a number of abundances $(\mathrm{C}, \mathrm{N}, \mathrm{O}$, plus n-capture elements) were derived by fitting relevant features manually.
Abundances for Na I, MgI, Si I, Ca I, Sc II, Ti I, Ti II, CrI, Mn I, Fe I, Fe II, Co I, Ni I, and Zn I were obtained by MyGIsFOS. Features for C I, N I, O I, and Eu II were fitted by means of FitProfile (Thygesen et al. 2016). All the remaining abundances were derived using MOOG (Sneden 1973, version 2014).

Table 2 lists the features used for the abundances measured with MOOG. Following Appendix A of Sbordone et al. (2015), details of the synthetic fits for all the lines measured by MyGIsFOS are made available at the CDS. Given the close affinity between MyGIsFOS and FitProfile, the lines we fit with the latter have been included as well.

- Carbon, nitrogen, and oxygen: The carbon abundance was derived by fitting the $\mathrm{CH} G$ band. From the range between $422.9 \mathrm{~nm}$ and $423.2 \mathrm{~nm}$, we also derived ${ }^{12} \mathrm{C} /{ }^{13} \mathrm{C}$. The nitrogen abundance was derived by fitting four $\mathrm{CN}$ features around $648 \mathrm{~nm}$, accounting for the $\mathrm{C}$ and $\mathrm{O}$ abundances in the synthesis. The oxygen abundance was derived from the $630 \mathrm{~nm}[\mathrm{O} \mathrm{I}]$ line. The blue wing of the line is marginally affected by a telluric line that does not compromise the fit, as has been verified by means of Molecfit (Smette et al. 2015; Kausch et al. 2015).

- Strontium: the only viable Sr II line in the spectrum is at $407.77 \mathrm{~nm}$, and it is extremely strong, to the point that it exceeds saturation and enters the damping part of the curve of growth. However, the wings are strongly blended. A manual fit, shown in Fig. 3, allows us to derive the abundance quoted here, but we do not trust the estimate to better than \pm 0.5 dex (the abundance difference of the two fits plotted around the best fit in Fig. 3).

- Europium: three Eu II lines were analyzed in J1907. For all we adopted the Lawler et al. (2001) atomic data, hyperfine and isotopic splitting. The bluest, at $412.960 \mathrm{~nm}$, is heavily saturated and strongly blended. It provides a tentative fit at $[\mathrm{Eu} / \mathrm{Fe}]=+0.7$. The $643.764 \mathrm{~nm}$ line is much weaker but provides an excellent fit at $[\mathrm{Eu} / \mathrm{Fe}]=+0.97$. This line is blended with a potentially problematic Si I line at $643.770 \mathrm{~nm}$. Jacobson \& Friel (2013) derived an astrophysical $\log g f$ of -2.3 for this line. In our line list, the transition is included with a higher $\log g f(-2.053)$, but even so, the line is extremely weak as a result of the low metallicity of the star and does not affect the Eu line. The $664.506 \mathrm{~nm}$ line, finally, is one of the most frequently used lines to determine Eu abundances, and it appears isolated and well fit, giving $[\mathrm{Eu} / \mathrm{Fe}]=+1.08$. Because the uncertainty on Eu II $412.960 \mathrm{~nm}$ is high, we rejected this line and only used the transitions at 643 and $664 \mathrm{~nm}$.

In Table 3 the final abundances are listed together with the assumed solar abundances and $[\mathrm{X} / \mathrm{H}]$ and $[\mathrm{X} / \mathrm{Fe}]$ ratios. This set of solar abundances is that of Caffau et al. (2011) or Lodders et al. (2009) for all elements not present in Caffau et al. (2011). Where multiple lines were used, the " \pm " columns list the $1 \sigma$ dispersion around the average for $[\mathrm{X} / \mathrm{H}]$, and the propagation accounting for the $[\mathrm{Fe} \mathrm{I} / \mathrm{H}]$ or $[\mathrm{Fe} I \mathrm{II} / \mathrm{H}]$ (for the $[\mathrm{OI}]$ $630.03 \mathrm{~nm}$ line, and ionized species) dispersion for the $[\mathrm{X} / \mathrm{Fe}]$ values. Together with the abundances listed in Table 3 , we also derived a carbon isotopic ratio of ${ }^{12} \mathrm{C} /{ }^{13} \mathrm{C}=12$.

The ${ }^{12} \mathrm{C} /{ }^{13} \mathrm{C}$ ratio is generally difficult to interpret in CEMP-s and $-\mathrm{r} / \mathrm{s}$, where carbon enhancement is believed to be due to mass accretion of heavily $\mathrm{C}$-enhanced ejecta from an AGB primary. $\mathrm{AGB}$ ejecta are believed to be very rich in ${ }^{12} \mathrm{C}$ (in our models, ${ }^{12} \mathrm{C} /{ }^{13} \mathrm{C}$ is comprised between 800 and 7600 ), while observed ratios in CEMP-s and -r/s are much lower (see Bisterzo et al. 2011, where values compiled from $\sim 50$ stars span values of $\left.4 \leq{ }^{12} \mathrm{C} /{ }^{13} \mathrm{C} \leq 90\right)$. This is due to the dilution of the AGB ejecta within the secondary convective envelope, which is poorer in ${ }^{12} \mathrm{C}$. However, a quantitative prediction is complicated by the unknown degree of dilution, the variations in ${ }^{13} \mathrm{C}$ abundance 
Table 3. Abundances for J1907.

\begin{tabular}{|c|c|c|c|c|c|c|c|}
\hline Ion & $N_{\text {lin }}$ & $\mathrm{A}(\mathrm{X})_{\odot}$ & $\mathrm{A}(\mathrm{X})$ & {$[\mathrm{X} / \mathrm{H}]$} & \pm & {$[\mathrm{X} / \mathrm{Fe}]$} & \pm \\
\hline $\mathrm{CI}_{\mathrm{I}}$ & 1 & 8.50 & 8.08 & -0.42 & 0.17 & 1.13 & 0.24 \\
\hline $\mathrm{NI}_{\mathrm{I}}$ & 4 & 7.86 & 6.82 & -1.04 & 0.21 & 0.51 & 0.26 \\
\hline O I & 1 & 8.76 & 7.61 & -1.15 & 0.17 & 0.40 & 0.24 \\
\hline $\mathrm{Na} I$ & 1 & 6.30 & 4.37 & -1.93 & 0.17 & -0.38 & 0.24 \\
\hline Mg I & 1 & 7.54 & 6.28 & -1.26 & 0.17 & 0.29 & 0.24 \\
\hline $\mathrm{Si} \mathrm{I}$ & 2 & 7.52 & 6.12 & -1.40 & 0.08 & 0.15 & 0.18 \\
\hline $\mathrm{Ca} \mathrm{I}$ & 9 & 6.33 & 5.09 & -1.24 & 0.16 & 0.32 & 0.23 \\
\hline Sc II & 7 & 3.10 & 1.70 & -1.40 & 0.16 & 0.15 & 0.23 \\
\hline Ti I & 6 & 4.90 & 3.60 & -1.30 & 0.18 & 0.25 & 0.24 \\
\hline Ti II & 3 & 4.90 & 3.44 & -1.46 & 0.48 & 0.09 & 0.51 \\
\hline Cr I & 4 & 5.64 & 4.04 & -1.60 & 0.23 & -0.05 & 0.28 \\
\hline Mn I & 6 & 5.37 & 3.62 & -1.75 & 0.50 & -0.19 & 0.52 \\
\hline $\mathrm{Fe} I$ & 51 & 7.52 & 5.97 & -1.55 & 0.17 & - & - \\
\hline Fe II & 10 & 7.52 & 5.97 & -1.55 & 0.16 & - & - \\
\hline Co I & 1 & 4.92 & 3.19 & -1.73 & 0.17 & -0.18 & 0.24 \\
\hline Ni I & 2 & 6.23 & 4.67 & -1.56 & 0.22 & -0.01 & 0.28 \\
\hline $\mathrm{Zn}$ I & 1 & 4.62 & 3.87 & -0.75 & 0.17 & 0.80 & 0.24 \\
\hline Sr II & 1 & 2.92 & 1.84 & -1.08 & 0.50 & 0.47 & 0.52 \\
\hline Y II $_{\text {II }}$ & 3 & 2.21 & 1.13 & -1.08 & - & 0.47 & 0.08 \\
\hline $\mathrm{ZrI}$ & 1 & 2.58 & 1.98 & -0.60 & 0.17 & 0.95 & 0.24 \\
\hline Zr II & 1 & 2.58 & 1.93 & -0.65 & 0.17 & 0.90 & 0.23 \\
\hline Ba II & 1 & 2.18 & 2.03 & -0.15 & 0.17 & 1.40 & 0.23 \\
\hline La II & 3 & 1.10 & 0.83 & -0.27 & 0.17 & 1.28 & 0.23 \\
\hline $\mathrm{Ce} \mathrm{I}$ & 1 & 1.58 & 1.43 & -0.15 & 0.17 & 1.40 & 0.24 \\
\hline Ce II & 5 & 1.58 & 1.30 & -0.28 & - & 1.27 & 0.06 \\
\hline Pr II & 3 & 0.72 & 0.40 & -0.32 & - & 1.23 & 0.06 \\
\hline Nd II & 9 & 1.42 & 1.19 & -0.23 & - & 1.32 & 0.11 \\
\hline Sm II & 3 & 1.00 & 0.66 & -0.34 & 0.13 & 1.21 & 0.20 \\
\hline Eu II & 2 & 0.52 & 0.00 & -0.52 & 0.08 & 1.03 & 0.17 \\
\hline Gd II & 1 & 1.07 & 0.67 & -0.40 & 0.17 & 1.15 & 0.23 \\
\hline Dy II & 1 & 1.10 & 0.75 & -0.35 & 0.17 & 1.20 & 0.23 \\
\hline
\end{tabular}

Notes. See Sect. 3 for details. Error for species with a single measured line are estimated according to Appendix A.

depending on the secondary evolutionary stage, and the generally uncertain theoretical predictions on these ${ }^{13} \mathrm{C}$ abundances, where additional mixing seems to be required to fit observations (Busso et al. 2010).

\section{Discussion}

In Fig. 1 we plot $[\alpha / \mathrm{Fe}]$ versus $[\mathrm{Fe} / \mathrm{H}]$ for $\mathrm{J} 1907$ together with literature Milky Way (MW) samples, other Sgr dSph stars, and mean values for globular clusters associated with Sgr dSph. In this respect, J1907 behaves as a typical member of the metal-poor population that dominates the outskirts of Sgr dSph: the very low $[\alpha / \mathrm{Fe}]$ and high $[\mathrm{Fe} / \mathrm{H}]$ observed in the central part of the galaxy (Sbordone et al. 2007; Tolstoy et al. 2009; McWilliam et al. 2013; Hasselquist et al. 2017) give way to higher levels of $\alpha$-enhancement, with Sgr dSph being indistinguishable from the MW halo for metallicity below $[\mathrm{Fe} / \mathrm{H}] \sim-2$ (Sbordone et al. 2015).

However, the abundances of J1907 allow us to classify it as a typical CEMP-r/s (or CEMP-i) star, and to the best of our knowledge, it is the first star of this kind identified in Sgr dSph.

The carbon abundance of J1907, plotted in Figs. 2 and 4, places it firmly in the "high-carbon band" defined by Spite et al. (2013) and Bonifacio et al. (2018) or the "Group I" defined by Yoon et al. (2016). Its [Sr/Ba] ratio also supports a CEMP-r/s classification according to Hansen et al. (2019) (see Fig. 5), and the same is true according to the Beers \& Christlieb (2005) classification scheme, with an $[\mathrm{Eu} / \mathrm{Fe}]=+1.03$, and consequently $[\mathrm{Ba} / \mathrm{Eu}]=+0.37$. Every measured element with atomic number $\geq 39$ (Y) shows enhancements close to or exceeding 1 dex with respect to iron $([\mathrm{X} / \mathrm{Fe}])$.

In Fig. 6 we plot $[\mathrm{Dy} / \mathrm{Ba}]$ and $[\mathrm{Dy} / \mathrm{Eu}]$ against metallicity. Dysprosium abundances are rare in the literature. Out of the stars with n-capture enhancement (CEMP-r, $-s,-r / s$ ) analyzed or collected from literature in Allen et al. (2012), a total of 19 have Dy abundances. To these we added 21 stars extracted from the SAGA database (Suda et al. 2011) that were originally published in Jonsell et al. (2006), Behara et al. (2010), Cui et al. (2013), Placco et al. (2013), Roederer et al. (2014), Hansen et al. (2015), Hollek et al. (2015), Placco et al. (2015), Jorissen et al. (2016), and Gull et al. (2018). It is common in the literature to study the $[\mathrm{La} / \mathrm{Eu}]$ ratios of these stars to determine their relative $s$-process and/or $r$-process enrichments (see, e.g., Roederer et al. 2010). In order to perform this classification, we can alternatively use dysprosium surface abundances. The left panel of Fig. 6 clearly shows that CEMP-s stars have the lowest $[\mathrm{Dy} / \mathrm{Ba}]$ values (and CEMP-r stars the highest values). Moreover, we can further refine this analysis by comparing dysprosium and europium, which are both typical $r$-process elements $(85 \%$ and $95 \%$ of their solar abundances, respectively; see Prantzos et al. 2020). Thus, the expected [Dy/Eu] spread between different classes of CEMP stars should be lower, as demonstrated in the right panel of Fig. 6. Neither Fig. 4 nor 6 show signs that the n-capture nucleosynthesis that affected J1907 is remarkably different from that in stars belonging to the MW.

In Fig. 7 we compare the abundance pattern in J1907 with a number of theoretical enrichment yields. In particular, we explore two completely different nucleosynthesis paths: a case with contributions from the $s$-process and $r$-process (for reviews see Straniero et al. 2006 and Cowan et al. 2019), and a case characterized only by the $i$-process. In Fig. 7 we report different models:

1. Dotted magenta curve: a neutron star merger (NSM) final surface element distribution (derived from element yields obtained using the SKYNET code with $Y_{e}=0.1$; Lippuner \& Roberts 2017), normalized to the J1907 europium overabundance, which is assumed as a representative element for the $r$-process.

2. Dashed green curve: final surface element distribution of a low-mass low-metallicity AGB star $\left(M=2 M_{\odot},[\mathrm{Fe} / \mathrm{H}]=-1.67\right.$; Cristallo et al. 2015), normalized to the J1907 lanthanum overabundance, which is assumed as a representative element for the $s$-process.

3. Solid dark curve: a combination of items 1 and 2, in which we assigned to each element the maximum abundance between the NSM and the AGB curves. This scenario includes pollution from two different sources: an AGB star providing the $s$-process enrichment, and an NSM supplying elements typical of the $r$-process. This hypothesis has been widely explored in the past (Bisterzo et al. 2010, 2011, 2012).

4. Solid blue curve: $i$-process nucleosynthesis resulting from RAWDs (Denissenkov et al. 2019) with $[\mathrm{Fe} / \mathrm{H}]=-1.55$, normalized to the J1907 europium overabundance.

Interpretation of the observed abundance pattern of J1907 is not straightforward, as is often the case for CEMP-r/s stars. Before we proceed with this analysis, however, some important caveats have to be set out.

Starting from AGB stars, it is worth to highlight that we find negligible variations by changing the metallicity (from 


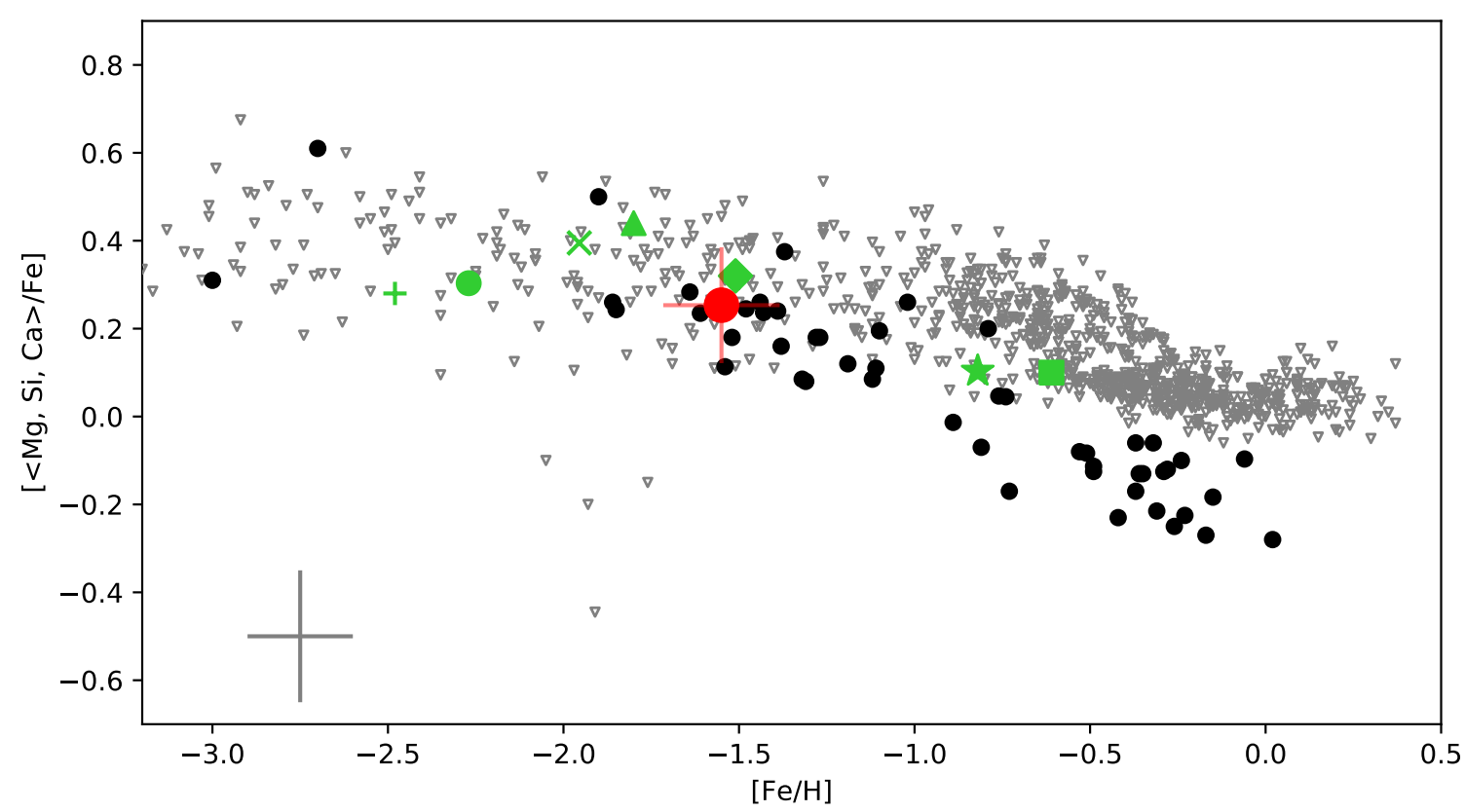

Fig. 1. $[\alpha / \mathrm{Fe}]$ vs. $[\mathrm{Fe} / \mathrm{H}]$ for $\mathrm{J} 1907$ (large red circle with error bars), compared to relevant samples. Small open gray triangles show MW stars (Venn et al. 2004; Reddy et al. 2006). Black filled circles represent the Sgr dSph main-body stars (Monaco et al. 2005; Sbordone et al. 2007, and in prep.; Hansen et al. 2018a). Green large symbols show the average values for globular clusters associated with Sgr dSph: the square is Ter 7; the star is Pal 12; the diamond is NGC 6715; the triangle is Arp 2; the X is NGC 5634; the circle is Ter 8; and the cross is NGC 5053 (Sbordone et al. 2005, 2015; Cohen 2004; Carretta et al. 2010, 2014; Mottini et al. 2008). [ $\alpha / \mathrm{Fe}]$ is here defined as the simple average of $[\mathrm{Mg} / \mathrm{Fe}],[\mathrm{Si} / \mathrm{Fe}]$, and $[\mathrm{Ca} / \mathrm{Fe}]$, or any subsample of them available for each specific star. A reference error bar of \pm 0.15 dex on both axes is also plotted.

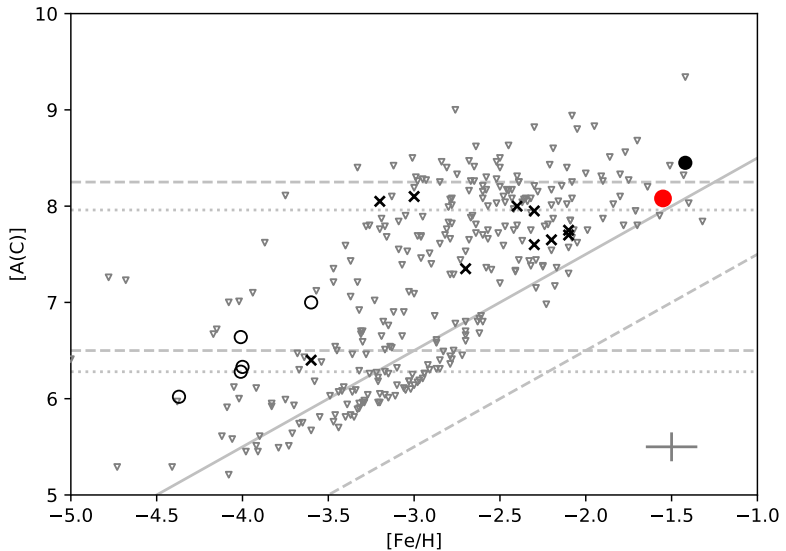

Fig. 2. $\mathrm{A}(\mathrm{C})$ vs. $[\mathrm{Fe} / \mathrm{H}]$ for $\mathrm{J} 1907$ (filled red circle) vs. different literature samples from Yoon et al. (2016, gray triangles), Hansen et al. (2019, black X), Bonifacio et al. (2018, open black circles), and star SDSS J100714+160154 from Frebel et al. (2014, filled black circle). Horizontal lines describe the high- and low-carbon band according to Spite et al. (2013, dashed) or Yoon et al. (2016, dotted). Diagonal lines are $[\mathrm{C} / \mathrm{Fe}]=0$ (dashed) and +1.0 (continuous). All comparison samples, except for SDSS J100714+160154, belong to the MW.

$[\mathrm{Fe} / \mathrm{H}]=-1.67$ to $[\mathrm{Fe} / \mathrm{H}]=-1.18$, the nearest more metal-rich model available in the FRUITY database ${ }^{7}$; Cristallo et al. 2015) or the initial mass $\left(1.5 \leq M / M_{\odot} \leq 3.0\right)$. This is proven by Fig. 8, where FRUITY models for different masses and metallicities are reported. Distributions with the label "norm" were normalized to the lanthanum abundance of J1907. Models with initial masses between $2 M_{\odot}$ and $3 M_{\odot}$ show almost the same distribution: this is because at these metallicities, both the light-s ( $l s$ elements:

\footnotetext{
7 http://fruity.oa-abruzzo.inaf.it/
}

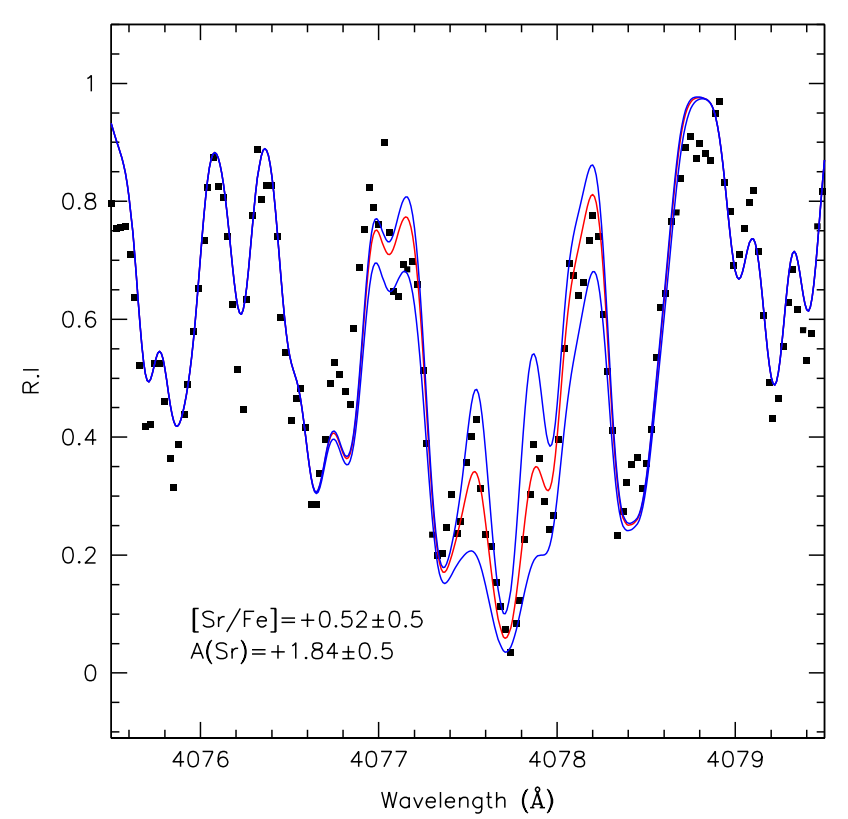

Fig. 3. Fit of the $407.77 \mathrm{~nm} \mathrm{Sr}$ II feature: The best fit, $\mathrm{A}(\mathrm{Sr})=1.84 \pm 0.5$, is represented by the red curve, while the two blue lines represent models corresponding to \pm 0.5 dex.

Sr-Y-Zr) and heavy-s ( $h s$ elements: Ba-La-Ce-Pr-Nd) elements are saturated. The only appreciable variations are found for lead, which has not been measured in our star (see below). It has to be stressed that the $1.3 M_{\odot}$ and the $4.0 M_{\odot}$ models are unable to attain the enrichment level of heavy-s elements found in J1907. While for the $1.3 M_{\odot}$ a slight increase in the mixing efficiency could solve the problem, the situation for the $4.0 M_{\odot}$ (and for more massive models) is different. A higher production of heavy 


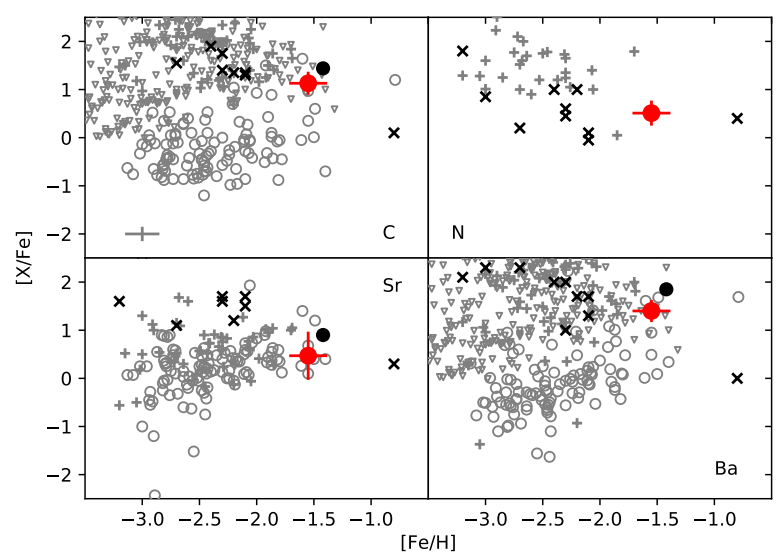

Fig. 4. $[\mathrm{X} / \mathrm{Fe}]$ vs. $[\mathrm{Fe} / \mathrm{H}]$ for $\mathrm{C}, \mathrm{N}, \mathrm{Sr}$, and $\mathrm{Ba}$. J1907 (filled red circle), Yoon et al. (2016, gray triangles), Hansen et al. (2019, black X), Hansen et al. (2018b, open gray circles), Allen et al. (2012, CEMP-s, -r, -s/r stars only, gray pluses), star SDSS J100714+160154 from Frebel et al. (2014, filled black circle). A 0.15 dex error cross is added for reference. All comparison samples, except for SDSS J100714+160154, belong to the MW.

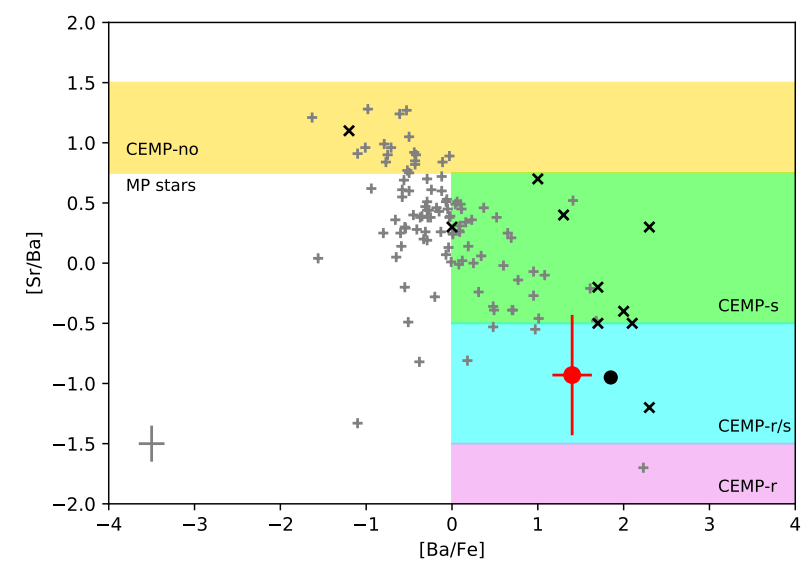

Fig. 5. $[\mathrm{Sr} / \mathrm{Ba}]$ vs. $[\mathrm{Ba} / \mathrm{Fe}]$ for $\mathrm{J} 1907$ (filled red circle), Hansen et al. (2019, black X), Hansen et al. (2018b, gray crosses), star SDSS J100714+160154 from Frebel et al. (2014, filled black circle). The areas corresponding to the CEMP classification proposed in Hansen et al. (2019) are shaded and labeled. A 0.15 dex error cross is added for reference.

elements would be compensated for by a poorer fit to light-s elements. Thus, we can reasonably exclude massive AGBs from the polluters of J1907.

The high carbon content in a relatively metal-rich star, the placement on the high-carbon band, and the strong overabundance of $s$-process elements all indicate that J1907 has been affected by mass transfer from an AGB companion. The star does not show straightforward radial velocity variations, but the limited coverage and precision makes this merely an "absence of evidence". The high Eu, Gd, and Dy abundances, however, are incompatible with an $s$-process-only source for neutroncapture elements. As for other CEMP-r/s stars, the abundances of $\mathrm{Eu}, \mathrm{Gd}$, and Dy can be explained with a pre-enrichment of the gas from which J1907 was formed. We hypothesize that this enrichment comes from an NSM. The infrared rebrightening of the electromagnetic transient AT2017gfo (Pian et al. 2017), following the gravitational wave event GW170817 (Abbott et al. 2017), proved that NSM events are characterized by a rich $r$-process nucleosynthesis (Watson et al. 2019). A key parameter

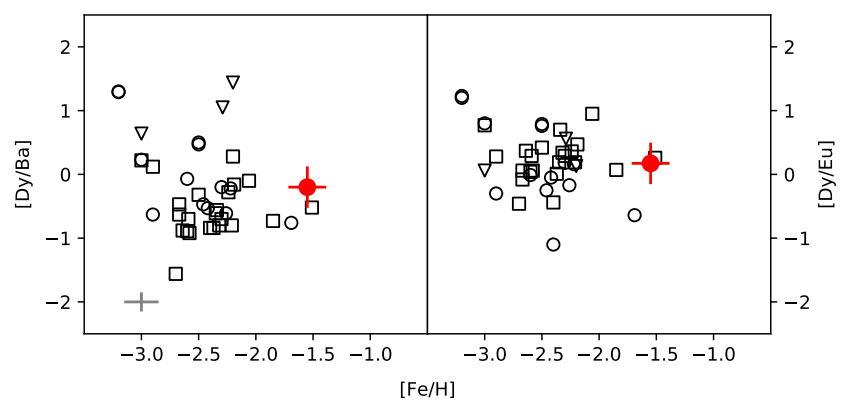

Fig. 6. $[\mathrm{Dy} / \mathrm{Ba}]$ and $[\mathrm{Dy} / \mathrm{Eu}]$ plotted against $[\mathrm{Fe} / \mathrm{H}]$ for $\mathrm{J} 1907$ (filled red circle) and literature CEMP-r (triangles), CEMP-s (squares), and CEMP-rs (circles). A 0.15 dex error cross is added for reference.

in these simulations is the electron-to-baryon ratio, the so-called $Y_{e}$. Depending on its initial value, completely different final distributions can be obtained (for a recent review, see Cowan et al. 2019). In our simulation we adopted a low $Y_{e}$, which is required to develop a full nucleosynthesis up to the third $r$-process peak. For the sake of clarity, it has to be noted that different components have been identified in a single NSM event, each of them marked by a different $Y_{e}$. Moreover, additional $r$-process polluters have been suggested, as magnetorotationally driven supernovae (Nishimura et al. 2017) or magnetars (Siegel et al. 2019): these stellar environments may provide heavy-element distributions similar to those of an NSM. A detailed comparison between these nucleosynthetic events is beyond the scope of our paper. We based our choice on the only (at the time of writing) proven source for the $r$-process nucleosynthesis, and we therefore assume that an NSM event provided the $r$-process component of $\mathrm{J} 1907$.

An alternative explanation for the combined pollution from the $s$-process and the $r$-process may come from the $i$-process. This process is thought to originate from the sudden injection of hydrogen into very hot layers (Cowan \& Rose 1977). One of the most uncertain ingredients characterizing $i$-process calculations is the stellar site hosting such a process. So far, three hypotheses have been explored with stellar evolutionary models:

1. Proton ingestion at the beginning of the thermally pulsing AGB phase of very low-metallicity low-mass stars: this process appears remote for $\mathrm{J} 1907$ because of its relatively high metallicity, which prevents the convective protons from being mixed in He-rich layers (see Cristallo et al. 2016 for details; see also Stancliffe et al. 2011 and Herwig et al. 2014 for very-low metallicity 3D hydrodynamical simulations). It has to be stressed that moderately metal-rich stars have been fit with $i$-process calculations (Roederer et al. 2016; Koch et al. 2019). However, these studies only included network calculations and did not clearly identify the stellar site hosting such a process.

2. Proton ingestion at the end of the thermally pulsing AGB phase (the so-called very late thermal pulse): this process has been proposed to explain the peculiar nucleosynthesis of Sakurai's object (Herwig et al. 2011). However, available calculations are for solar metallicity only.

3. RAWDs, which are able to simultaneously produce both $s$-process elements (such as lanthanum) and $r$-process elements (such as europium): simulations for these events are available at different metallicities (Denissenkov et al. 2019).

In addition to these stellar models, network calculations have been published by Hampel et al. (2016): even if performed at metallicities lower than J1907, they can be used to track its nucleosynthesis because of the saturation effect of the ls and 


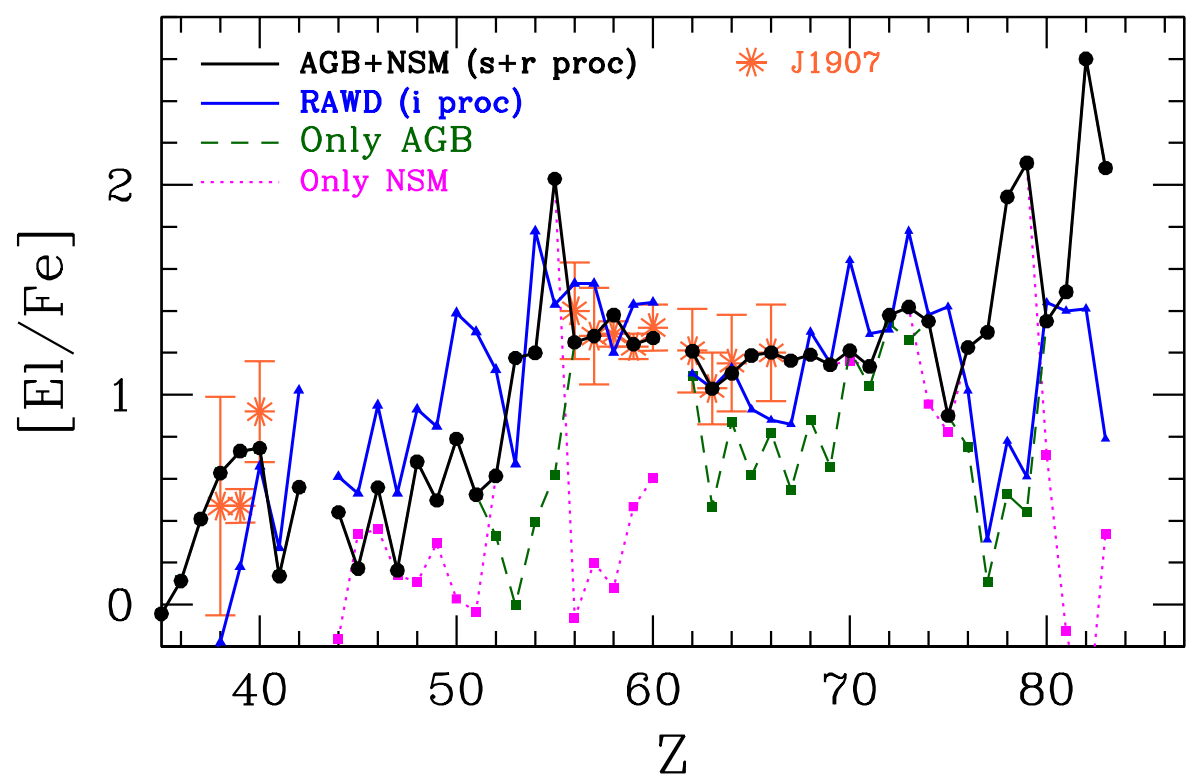

Fig. 7. Abundance pattern of J1907 compared with yields from a NMS (magenta dotted curve), an AGB star (green dashed curve), a combination of them (black solid curve) and RAWDs (blue solid curve). See text for details.

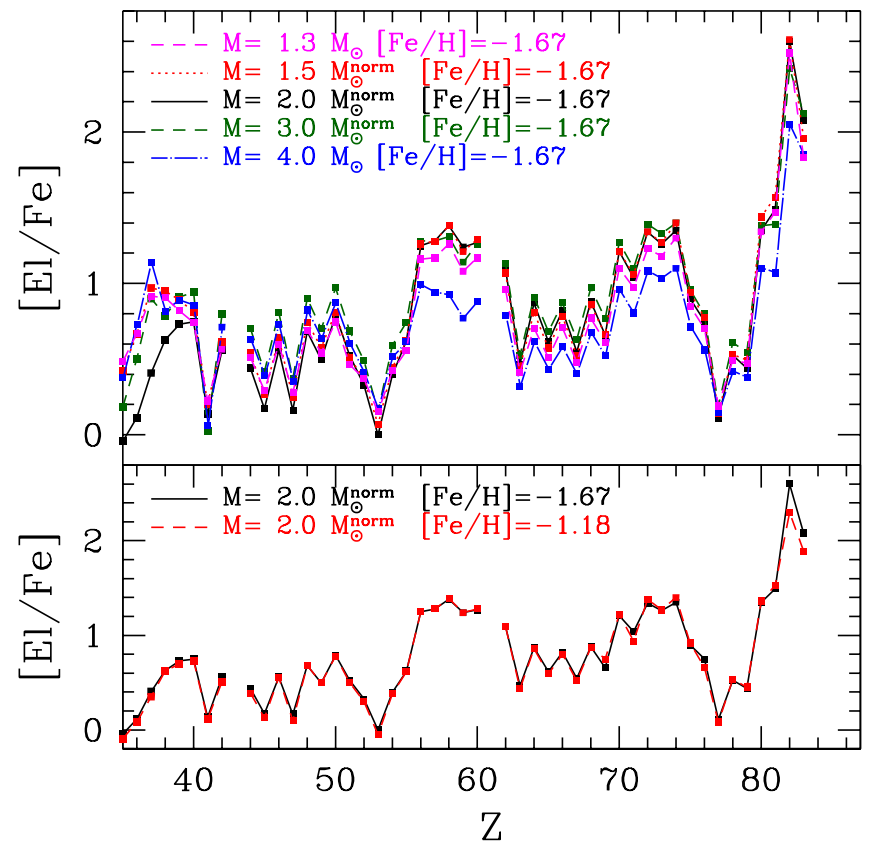

Fig. 8. FRUITY AGB models with different initial masses (upper panel) and at different metallicities (lower panel). See text for details.

the hs component of the $s$-process (see above). In Fig. 9 we report network calculations for different neutron densities (normalized to europium), and we compare them to the J1907 distribution. From a visual inspection, we can safely exclude the lowest $\left(10^{12} \mathrm{~cm}^{-3}\right)$ and the highest $\left(10^{15} \mathrm{~cm}^{-3}\right)$ neutron density cases. The first is not able to fit any of the hs elements, while the latter does not match ls elements and barium. Intermediate cases (i.e., between $10^{13} \mathrm{~cm}^{-3}$ and $10^{14} \mathrm{~cm}^{-3}$ ) provide a good enough fit, with the exclusion of gadolinium (this problem is shared with the calculation by Denissenkov et al. 2019, which also underproduces light-s elements). On the other hand, these network calculations provide a good fit to the large observed $[\mathrm{Zr} / \mathrm{Y}]$, which is difficult to obtain with $s$-process models.

In the comparison of Fig. 7, we opt for discussing only the distribution obtained with stellar evolutionary calculations, but

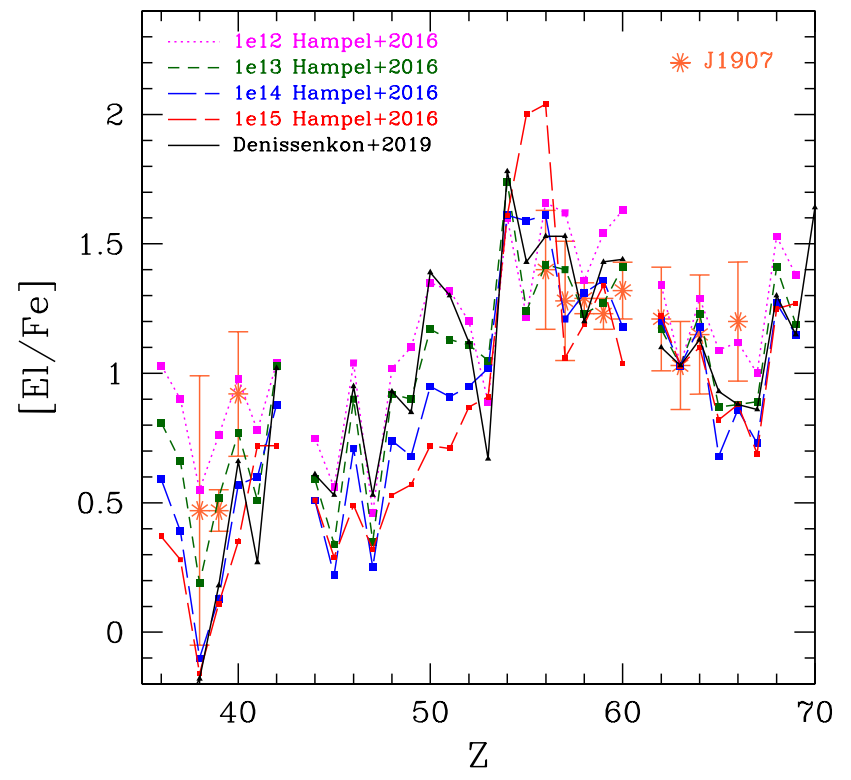

Fig. 9. $i$-process nucleosynthesis calculation (Hampel et al. 2016) for different neutron densities compared to J1907. The distribution at $[\mathrm{Fe} / \mathrm{H}]=-1.55$ from Denissenkov et al. (2019) is also reported. See text for details.

in the future, useful constraints can also be derived from the above-mentioned network calculations.

For the sake of clarity, it has to be stressed that both scenarios (RAWD vs. AGB+NSM) have difficulties to explain from a statistical point of view the CEMP populations observed in the halo of our Galaxy (Abate et al. 2016, even if this analysis was performed for lower metallicities). With the current element list, it is objectively difficult to distinguish between the two described scenarios. The situation would be completely different if at least one element belonging to the pairs $\mathrm{Pt}-\mathrm{Au}$ and $\mathrm{Pb}-\mathrm{Bi}$ were detected. For these elements, the $i$-process scenario shows definitely lower enhancements than the $s+r$ scenario, where they are largely abundant (the $\mathrm{Pt}-\mathrm{Au}$ pair due to a pre-existing $r$-process, and the $\mathrm{Pb}-\mathrm{Bi}$ couple from AGB pollution). Further observations dedicated to this star would be very useful. 
J1907 is fairly similar, from a chemical point of view, to SDSS J100714+160154, which was studied in the ultra-faint dwarf galaxy Segue 1 by Frebel et al. (2014), as we show in Figs. 2, 4, and 5. However, SDSS J100714+160154 shows more pronounced $s$-process enrichment (e.g., $[\mathrm{Sr} / \mathrm{Fe}]=0.9$, $[\mathrm{Zr} / \mathrm{Fe}]=1.4,[\mathrm{Ba} / \mathrm{Fe}]=1.85)$ and a measured $\mathrm{Pb}$ abundance, while $\mathrm{Pb}$ was not detected in the available spectrum of J1907. The two stars share a high [hs/ls] ratio ${ }^{8}$. On the other hand, $r$-process elements are less enhanced, with $[\mathrm{Eu} / \mathrm{Fe}]=0.7$. This would make it a CEMP-s star according to the Beers \& Christlieb (2005) classification, while the Hansen et al. (2019) classification would indicate it as a CEMPr/s (Fig. 5).

\section{Conclusions}

We presented a chemical analysis for the star GIU J190734.24315102.1 (J1907), a member of Sgr dSph. The star is a moderately metal-poor $([\mathrm{FeH}]=-1.55)$ giant, displaying strong carbon enhancement $([\mathrm{C} / \mathrm{Fe}]=+1.13)$, and $n$-capture enhancement of both $s$ - and $r$-process elements $([\mathrm{Ba} / \mathrm{Fe}]=+1.4$, $[\mathrm{Eu} / \mathrm{Fe}]=+1.0)$.

To the best of our knowledge, J1907 is the first CEMP-r/s detected in the Sgr dSph. The situation is less obvious in other dwarf galaxies of the Local Group.

- UMi K (Shetrone et al. 2001), Fornax 21 (Shetrone et al. 2003), and Sculptor 982 (Geisler et al. 2005) are the earliest reports we can find of $\mathrm{Ba}$ - and Eu-enhanced stars in Local Group dwarf spheroidal galaxies. Their heavy-element abundances would classify them as CEMP-r/s according to the Beers \& Christlieb (2005) scheme, but their carbon abundance has never been determined. Sculptor 982 is reported (Hill et al. 2019; Skúladóttir et al. 2020) to show very strong CN bands, a further hint in this direction.

- The aforementioned SDSS J100714+160154 presented in Frebel et al. (2014) is borderline between CEMP-s and CEMP$\mathrm{r} / \mathrm{s}$, to the extent that a sharp threshold at $[\mathrm{Ba} / \mathrm{Eu}]=0.5$ makes physical sense.

- Chiti et al. (2018), in a search for CEMP stars in the Sculptor dSph, identified 11 candidate CEMP-s / r/s, based on their high $\mathrm{C}$ abundance, placing them in the "high-carbon band" (see also Fig. 2). Only 3 of them have Ba abundances, and only one of these shows a significant Ba overabundance. In none of them are $\mathrm{Sr}$ or Eu abundances measured.

With the current available models, J1907 appears to be best explained as the product of a mass exchange from an AGB companion within a binary system that is pre-enriched at high concentration by the yields of an NS-NS merger.

It seems unlikely that this r-enrichment event only affected one star that thus also underwent AGB mass transfer. Other $r$-enriched stars should be present among the Sgr dSph stars of similar metallicity that formed together with J1907. Because the star is placed at the extreme end of the major axis of the main body of the Sgr dSph, a good number of such stars might since have been stripped by the interaction with the MW, and might currently reside in the Sgr dSph stream.

Acknowledgements. This paper is dedicated to the dear memory of the late Fiorella Castelli, whose work in the modeling and analysis of stellar atmospheres has been a fundamental guidance for us. The authors thank Diego Vescovi for help in the use of the SKYNET code. EC and PB gratefully acknowledge support from the French National Research Agency (ANR) funded project "Pristine" (ANR-18-CE31-0017). EC and PB are thankful to ESO - Santi-

\footnotetext{
8 This ratio defines the enrichment of the second peak of the $s$-process (hs elements) with respect to the first peak (ls elements), and it is defined as $[\mathrm{hs} / \mathrm{ls}]=[\mathrm{hs} / \mathrm{Fe}]-[\mathrm{ls} / \mathrm{Fe}]$ in the usual spectroscopic notation.
}

ago for hosting them during the preparation of this manuscript. SV gratefully acknowledges the support provided by Fondecyt reg. n. 1170518. This work has made use of data from the European Space Agency (ESA) mission Gaia (https: //www. cosmos.esa.int/gaia), processed by the Gaia Data Processing and Analysis Consortium (DPAC, https://www. cosmos.esa.int/web/ gaia/dpac/consortium). This work has made use of data from APOGEE, part of SDSS IV. The SDSS web site is www.sdss.org.

\section{References}

Abate, C., Stancliffe, R. J., \& Liu, Z.-W. 2016, A\&A, 587, A50 Abate, C., Pols, O. R., \& Stancliffe, R. J. 2018, A\&A, 620, A63

Abbott, B. P., LIGO Scientific Collaboration, \& Virgo Collaboration 2017, Phys. Rev. Lett., 119, 161101

Allen, D. M., Ryan, S. G., Rossi, S., Beers, T. C., \& Tsangarides, S. A. 2012, A\&A, 548, A34

Arentsen, A., Starkenburg, E., Shetrone, M. D., et al. 2019, A\&A, 621, A108

Baumgardt, H., Hilker, M., Sollima, A., \& Bellini, A. 2019, MNRAS, 482, 5138

Beers, T. C., \& Christlieb, N. 2005, ARA\&A, 43, 531

Behara, N. T., Bonifacio, P., Ludwig, H. G., et al. 2010, A\&A, 513, A72

Bellazzini, M., Ibata, R. A., Chapman, S. C., et al. 2008, AJ, 136, 1147

Bisterzo, S., Gallino, R., Straniero, O., Cristallo, S., \& Käppeler, F. 2010, MNRAS, 404, 1529

Bisterzo, S., Gallino, R., Straniero, O., Cristallo, S., \& Käppeler, F. 2011, MNRAS, 418, 284

Bisterzo, S., Gallino, R., Straniero, O., Cristallo, S., \& Käppeler, F. 2012, MNRAS, 422, 849

Bonifacio, P., Caffau, E., Spite, M., et al. 2018, A\&A, 612, A65

Busso, M., Palmerini, S., Maiorca, E., et al. 2010, ApJ, 717, L47

Caffau, E., Ludwig, H. G., Steffen, M., Freytag, B., \& Bonifacio, P. 2011, Sol. Phys., 268, 255

Caffau, E., Koch, A., Sbordone, L., et al. 2013, Astron. Nachr., 334, 197

Caffau, E., Gallagher, A. J., Bonifacio, P., et al. 2018, A\&A, 614, A68

Carretta, E., Bragaglia, A., Gratton, R. G., et al. 2010, A\&A, 520, A95

Carretta, E., Bragaglia, A., Gratton, R. G., et al. 2014, A\&A, 561, A87

Castelli, F. 2005, Mem. Soc. Astron. It., 8, 25

Cayrel, R. 1988, The Impact of Very High S/N Spectroscopy on Stellar Physics, ed. G. Cayrel de Strobel, \& M. Spite, IAU Symp., 132, 345

Chiti, A., Simon, J. D., Frebel, A., et al. 2018, ApJ, 856, 142

Cohen, J. G. 2004, AJ, 127, 1545

Cowan, J. J., \& Rose, W. K. 1977, ApJ, 212, 149

Cowan, J.J., Sneden, C., Lawler, J. E., et al. 2019, Rev. Mod. Phys. submitted [arXiv:1901.01410]

Cristallo, S., Piersanti, L., Straniero, O., et al. 2011, ApJS, 197, 17

Cristallo, S., Straniero, O., Piersanti, L., \& Gobrecht, D. 2015, ApJS, 219, 40

Cristallo, S., Karinkuzhi, D., Goswami, A., Piersanti, L., \& Gobrecht, D. 2016, ApJ, 833, 181

Cui, W. Y., Sivarani, T., \& Christlieb, N. 2013, A\&A, 558, A36

Dekker, H., D’Odorico, S., Kaufer, A., Delabre, B., \& Kotzlowski, H. 2000,

Optical and IR Telescope Instrumentation and Detectors, eds. M. Iye, \& A. F. Moorwood, 4008, 534

Denissenkov, P. A., Herwig, F., Woodward, P., et al. 2019, MNRAS, 488, 4258

François, P., Depagne, E., Hill, V., et al. 2007, A\&A, 476, 935

Frebel, A., Simon, J. D., \& Kirby, E. N. 2014, ApJ, 786, 74

Gaia Collaboration (Helmi, A., et al.) 2018, A\&A, 616, A12

Geisler, D., Smith, V. V., Wallerstein, G., Gonzalez, G., \& Charbonnel, C. 2005 , AJ, 129, 1428

Giuffrida, G., Sbordone, L., Zaggia, S., et al. 2010, A\&A, 513, A62

Gull, M., Frebel, A., Cain, M. G., et al. 2018, ApJ, 862, 174

Hampel, M., Stancliffe, R. J., Lugaro, M., \& Meyer, B. S. 2016, ApJ, 831, 171

Hansen, T., Hansen, C. J., Christlieb, N., et al. 2015, ApJ, 807, 173

Hansen, C. J., El-Souri, M., Monaco, L., et al. 2018a, ApJ, 855, 83

Hansen, T. T., Holmbeck, E. M., Beers, T. C., et al. 2018b, ApJ, 858, 92

Hansen, C. J., Hansen, T. T., Koch, A., et al. 2019, A\&A, 623, A128

Harris, W. E. 1996, AJ, 112, 1487

Hasselquist, S., Shetrone, M., Smith, V., et al. 2017, ApJ, 845, 162

Herwig, F., Pignatari, M., Woodward, P. R., et al. 2011, ApJ, 727, 89

Herwig, F., Woodward, P. R., Lin, P.-H., Knox, M., \& Fryer, C. 2014, ApJ, 792,

L3

Hill, V., Skúladóttir, Á., Tolstoy, E., et al. 2019, A\&A, 626, A15

Hollek, J. K., Frebel, A., Placco, V. M., et al. 2015, ApJ, 814, 121

Jacobson, H. R., \& Friel, E. D. 2013, AJ, 145, 107

Jonsell, K., Barklem, P. S., Gustafsson, B., et al. 2006, A\&A, 451, 651

Jorissen, A., Hansen, T., Van Eck, S., et al. 2016, A\&A, 586, A159

Käppeler, F., Gallino, R., Bisterzo, S., \& Aoki, W. 2011, Rev. Mod. Phys., 83, 157 
Kausch, W., Noll, S., Smette, A., et al. 2015, A\&A, 576, A78

Koch, A., Reichert, M., Hansen, C. J., et al. 2019, A\&A, 622, A159

Kurucz, R. L. 2005, Mem. Soc. Astron. It., 8, 14

Lawler, J. E., Wickliffe, M. E., den Hartog, E. A., \& Sneden, C. 2001, ApJ, 563, 1075

Le Fèvre, O., Saisse, M., Mancini, D., et al. 2003, Instrument Design and Performance for Optical/Infrared Ground-based Telescopes, eds. M. Iye, \& A. F. M. Moorwood, Proc. SPIE, 4841, 1670

Lippuner, J., \& Roberts, L. F. 2017, ApJS, 233, 18

Lodders, K., Palme, H., \& Gail, H. P. 2009, Landolt Börnstein, 4B, 712

Lucatello, S., Tsangarides, S., Beers, T. C., et al. 2005, ApJ, 625, 825

Majewski, S. R., Hasselquist, S., Łokas, E. L., et al. 2013, ApJ, 777, L13

McWilliam, A., Wallerstein, G., \& Mottini, M. 2013, ApJ, 778, 149

Monaco, L., Bellazzini, M., Bonifacio, P., et al. 2005, A\&A, 441, 141

Mottini, M., Wallerstein, G., \& McWilliam, A. 2008, AJ, 136, 614

Nishimura, N., Takiwaki, T., \& Thielemann, F.-K. 2015, ApJ, 810, 109

Nishimura, N., Sawai, H., Takiwaki, T., Yamada, S., \& Thielemann, F. K. 2017, ApJ, 836, L21

Pasquini, L., Avila, G., Allaert, E., et al. 2000, Optical and IR Telescope Instrumentation and Detectors, eds. M. Iye, \& A. F. Moorwood, 4008, 129

Pian, E., D'Avanzo, P., Benetti, S., et al. 2017, Nature, 551, 67

Placco, V. M., Frebel, A., Beers, T. C., et al. 2013, ApJ, 770, 104

Placco, V. M., Beers, T. C., Ivans, I. I., et al. 2015, ApJ, 812, 109

Prantzos, N., Abia, C., Cristallo, S., Limongi, M., \& Chieffi, A. 2020, MNRAS, 491, 1832

Reddy, B. E., Lambert, D. L., \& Allende Prieto, C. 2006, MNRAS, 367, 1329

Roederer, I. U., Cowan, J. J., Karakas, A. I., et al. 2010, ApJ, 724, 975

Roederer, I. U., Preston, G. W., Thompson, I. B., et al. 2014, AJ, 147, 136

Roederer, I. U., Karakas, A. I., Pignatari, M., \& Herwig, F. 2016, ApJ, 821, 37

Sbordone, L. 2005, Mem. Soc. Astron. It., 8, 61

Sbordone, L., Bonifacio, P., Castelli, F., \& Kurucz, R. L. 2004, Mem. Soc. Astron. It., 5, 93

Sbordone, L., Bonifacio, P., Marconi, G., Buonanno, R., \& Zaggia, S. 2005, A\&A, 437, 905

Sbordone, L., Bonifacio, P., Buonanno, R., et al. 2007, A\&A, 465, 815

Sbordone, L., Caffau, E., Bonifacio, P., \& Duffau, S. 2014, A\&A, 564, A109

Sbordone, L., Monaco, L., Moni Bidin, C., et al. 2015, A\&A, 579, A104

Shetrone, M. D., Côté, P., \& Sargent, W. L. W. 2001, ApJ, 548, 592

Shetrone, M., Venn, K. A., Tolstoy, E., et al. 2003, AJ, 125, 684

Siegel, D. M., Barnes, J., \& Metzger, B. D. 2019, Nature, 569, 241

Skúladóttir, Á, Hansen, C. J., Choplin, A., et al. 2020, A\&A, 634, A84

Smette, A., Sana, H., Noll, S., et al. 2015, A\&A, 576, A77

Sneden, C. A. 1973, Ph.D. Thesis, The University of Texas at Austin, USA

Spite, M., Caffau, E., Bonifacio, P., et al. 2013, A\&A, 552, A107

Stancliffe, R. J., Dearborn, D. S. P., Lattanzio, J. C., Heap, S. A., \& Campbell,

S. W. 2011, ApJ, 742, 121

Starkenburg, E., Shetrone, M. D., McConnachie, A. W., \& Venn, K. A. 2014 MNRAS, 441, 1217

Straniero, O., Gallino, R., \& Cristallo, S. 2006, Nucl. Phys. A, 777, 311

Suda, T., Yamada, S., Katsuta, Y., et al. 2011, MNRAS, 412, 843

Thielemann, F. K., Eichler, M., Panov, I. V., \& Wehmeyer, B. 2017, Ann. Rev. Nucl. Part. Sci., 67, 253

Thygesen, A. O., Sbordone, L., Ludwig, H.-G., et al. 2016, A\&A, 588, A66

Tolstoy, E., Hill, V., \& Tosi, M. 2009, ARA\&A, 47, 371

Trager, S. C., King, I. R., \& Djorgovski, S. 1995, AJ, 109, 218

Venn, K. A., Irwin, M., Shetrone, M. D., et al. 2004, AJ, 128, 1177

Watson, D., Hansen, C. J., Selsing, J., et al. 2019, Nature, 574, 497

Yoon, J., Beers, T. C., Placco, V. M., et al. 2016, ApJ, 833, 20

\section{Appendix A: Estimation of errors on atmosphere parameters and abundances}

Table 1 quotes MyGIsFOS internal parameters errors. They are computed as described below.

- During $T_{\text {eff }}$ calculation, the linear fit of the relationship between lower energy and abundance given by individual Fe I lines is computed, and $T_{\text {eff }}$ is iteratively adjusted until its slope is (very close to) zero (see Sbordone et al. 2014). This populates a list of $T_{\text {eff-slope pairs, against which a second-order }}$ polynomial is fit. When the final $T_{\text {eff }}$ is chosen, the error on the slope for that last fit is used together with this secondorder polynomial to determine which temperatures would correspond to slopes equal to final_slope-slope_error and final_slope+slope_error.

- Similarly, $V_{\text {turb }}$ is determined by iteratively finding the value that zeroes the slope of the relationship between the reduced equivalent width and abundance of individual $\mathrm{Fe}$ I lines. As the final value is found, the error of the slope and the stored $V_{\text {turb}} /$ slope pairs are used to determine the $V_{\text {turb }}$ uncertainty.

- Gravity is determined by enforcing equal average abundance to be given by Fe I and Fe II lines. The internal error (line-to-line dispersion) of Fe I and Fe II average abundances are summed in quadrature, and the uncertainty in gravity is determined by deriving the gravity that produces $\mathrm{A}(\mathrm{Fe} \mathrm{I})-\mathrm{A}(\mathrm{Fe} \mathrm{II})$ offsets equal to final_offset - quadrature_error and final_offset + quadrature_error.

Because the formal values of these uncertainties are sensitive to the way the parameters changed during the actual parametersearch iteration, we conservatively round uncertainties to $10 \mathrm{~K}$, $0.1 \mathrm{~km} \mathrm{~s}^{-1}$, and $0.1 \mathrm{~cm} \mathrm{~s}^{-2}$ for $T_{\text {eff }}, V_{\text {turb }}$, and $\log g$.

The quoted errors on abundances are estimated in different fashions.

- For abundances of species measured with MyGIsFOS and based on at least two lines, uncertainties on $[\mathrm{X} / \mathrm{H}]$ are lineto-line scatter, while the $[\mathrm{X} / \mathrm{Fe}]$ values are summed in quadrature with either Fe I or Fe II line-to-line scatter (see Sbordone et al. 2014, for details). The N I abundance, although determined with FitProfile, is based on multiple features and treated in the same way.

- Species measured with MOOG and based on multiple features also quote line-to-line scatter, however, because in this case, $[\mathrm{X} / \mathrm{Fe}]$ was measured directly, only the scatter on it is reported.

- Species based on a single feature (with the exception of Sr II, see above) do not allow directly probing the main sources of uncertainty. For isolated lines, the formal measurement uncertainty on the equivalent width, according to Cayrel \& Cayrel (1988), does not exceed 5-10\% for unsaturated lines at the typical $\mathrm{S} / \mathrm{N}$ of this spectrum, with a negligible $(0.04 \mathrm{dex}$, see also Caffau et al. 2013, Table 3 for a Monte Carlo test on simulated noise-injected spectra) effect on abundances. Line-to-line scatter in the best-sampled species (Fe I and Fe II) is around 0.16 dex, even on carefully selected lines. This underscores the well-known fact that abundance measurements are dominated by systematics (approximations on physics, uncertain atomic data, undetected or misestimated blends, and poor continuum placement in crowded regions). We therefore quote for all these species the Fe I line-to-line scatter as representative uncertainty on $[\mathrm{X} / \mathrm{Fe}]$, and then sum it in quadrature with either the Fe I or the Fe II line-to-line scatter for $[\mathrm{X} / \mathrm{Fe}]$.

We do not investigate the dependence of the abundances on the chosen atmosphere parameters here, however, as reported by François et al. (2007), the parameter sensitivity for stars of similar characteristics (BS 17569-049, Table 2 in François et al. 2007) is lower than the uncertainties we quote and can therefore be neglected. 